\title{
À Propos de deUX ObSERVATIONS d'helminthoses hépatiques SUR DES MARMOTTES, MARMOTA MONAX, INFECTÉES PAR LE VIRUS DE L'HÉPATITE VIRALE (WHV)
}

\author{
GEVREY J.*, BEUGNET F.* \& JAMARD C.**
}

Summary : NOTES ON TWO HEPATIC HELMINTHOSIS OF MARMOTTA MONAX INFECTED BY THE WOODCHUCK HePATtTIS Virus

Autopsy of two Woodchuck Hepatitis Virus (WHV) infected Woodchucks, Marmota monax, revealed the presence of two parasites in an hepatic localization, Taenia mustelae (Larvae) and Calodium hepaticum. The authors present the identification of the two parasites, based on the observation of cysticerci of Taenia mustelae, or on the observation of the eggs of C.hepaticum. They discuss the probable interaction between hepatic parasites and WHV infection.

KEY WORDS : Marmota monax, viral hepatitis, Taenia mustelae, Calodium hepaticum

\section{INTRODUCTION}

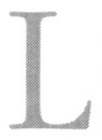

a marmotte américaine, Marmota monax, est utilisée comme modèle animal d'hépatite virale. Elle est en effet réceptive au virus WHV (Woodchuck Hepatitis virus), alors que la marmotte européenne, Marmota marmota, présente en France, ne l'est pas. A ce titre, des marmottes, séropositives ou séronégatives, sont importées à l'École Nationale Vétérinaire de Lyon, Unité Associée INSERM U-271. Elles proviennent du Northeastern Wildlife, une société spécialisée dans la commercialisation des marmottes (Northeastern Wildlife. PO Box 1000. South Plymouth, NY 13844).

Lors de l'autopsie de deux marmottes infectées par le WHV, des lésions parasitaires ont été notées. Les parasites identifiés sont Calodium hepaticum (syn. Capillaria bepatica) et la larve de Taenia mustelae. Ces observations indiquent que les animaux proviennent de captures dans le milieu sauvage, et posent le problème de la spécificité des lésions hépatiques observées.

\footnotetext{
* Unité d'Écologie Microbienne et Parasitaire, Département de Santé Publique Vétérinaire, École Nationale Vétérinaire de Lyon, 1, Av. Bourgelat, BP 83, Marcy-L'Étoile, F-69280.

${ }^{* *}$ INSERM U-271, École Nationale Vétérinaire de Lyon, 1, Av. Bourgelat, BP 83, Marcy-L'Étoile, F-69280.
}

\section{Résumé :}

Deux autopsies de marmottes américaines, Marmota monax, servant de modèle expérimental d'hépatite virale, ont permis l'identification de deux parasites à localisation hépatique, Taenia mustelae (larvae) (Cestoda: Taeniidae), et Calodium hepaticum (Nematoda : Enoplida: Capillariinae). Les auteurs présentent l'identification de ces parasites, basée sur l'observation des cysticerques dans le cas de Taenia mustelae, et sur celle des oeuts dans le cas de l'infestation par C. hepaticum. Le problème de l'inter-action possible entre l'infestation parasitaire à localisation hépatique et l'infection virale par le Woodchuck Hepatitis Virus reste posé.

MOTS CLÉS : Marmota monax, hépatite virale, Taenia mustelae, Calodium hepaticum.

\section{MATÉRIELS ET MÉTHODES}

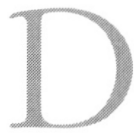

eux marmottes infectées par le WHV sont autopsiées, la première est morte en décembre 1994, du fait de l'infection virale ayant induit un hépatocarcinome, la seconde a été euthanasiée en octobre 1995.

Les viscères sont observés macroscopiquement. Les foies sont incisés, des fragments de $3 \mathrm{~mm}^{3}$ sont écrasés et dilacérés dans du chloral-lactophénol, puis observés à la loupe binoculaire, en vue de rechercher la présence de parasites, et particulièrement de Calodium hepaticum.

Des prélèvements de foie et de rein de $0,5 \mathrm{~cm}^{3}$ sont fixés dans le formol commercial dilué à $10 \%$ en vue de la réalisation de coupes histologiques. Une coloration Hémalun-Éosine est effectuée.

\section{RÉSULTATS}

MARMOtTe No 1 (décembre 1994)

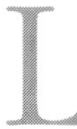

e foie présente un aspect et une consistance normaux. Une dizaine de " nodules " blanchâtres de 1 à $3 \mathrm{~mm}$ de diamètre sont observés en superficie et au sein du parenchyme hépatique. Ils sont formés d'une adventice renfermant une vésicule de 1,7 à $2 \mathrm{~mm}$ de diamètre. L'observation de cette vésicule indique qu'il s'agit d'un cysticerque : larve vésiculaire possédant une seule invagination céphalique. La dila- 
cération d'un cysticerque permet d'en effectuer la mensuration : le scolex est armé, muni d'un rostre d'environ $250 \mu \mathrm{m}$ de diamètre. Ce rostre est constitué de deux couronnes concentriques de crochets de petite dimension, en forme de poignards, 27 crochets pour la grande couronne, 23 pour la petite (photo 1). Ils mesurent 18 à $20 \mu \mathrm{m}$ de longueur pour ceux de la petite couronne, contre 20 à 22 pour ceux de la grande couronne. Ils ont un manche et une lame de tailles égales et une garde très épaisse. Des cysticerques sont également retrouvés dans les reins de ces marmottes. La taille des cysticerques et la morphologie des crochets est caractéristique de la larve de Taenia mustelae (Abuladze, 1970).

L'examen histologique permet l'observation d'un parenchyme hépatique normal et de coupes de cysticerques (photo 2). Les corpuscules calcaires, caractéristiques des cestodes, sont nettement visibles au sein des cysticerques après coloration. Dans les coupes histologiques, ils apparaissent sous forme de granules colorés en noir. L'épaisseur de la réaction conjonctive d'enkystement formant l'adventice doit être notée. La périphérie de l'adventice est formée par un infiltrat riche en plasmocytes et en polynucléaires éosinophiles.

\section{MARMOTTE $\mathrm{N}^{\circ} 2$ (octobre 1995)}

Le foie est décoloré et présente une consistance très ramollie. La dilacération, l'éclaircissement, puis l'observation du parenchyme mettent en évidence l'envahissement du tissu par des œufs de capillaires. Les nématodes ne peuvent être individualisés du parenchyme. Les oufs ont une coque striée, épaisse, et claire. Ils présentent à chaque extrémité un bouchon polaire aplati. Ils mesurent 48,4-55,8 × 29,2-31 $\mu \mathrm{m}$ (photo 3). La localisation hépatique et l'aspect des oufs est caractéristique d'une infestation par Calodium hepaticum (Bancroft, 1893; Gevrey et Chirol, 1978; Singleton et al., 1991).

L'examen histologique montre la présence de nombreux œufs de Calodium hepaticum au sein d'un parenchyme hépatique altéré (photo 4). Ce dernier est en partie lysé. Des zones riches en œufs alternent avec des plages de tissu sain, et des plages mal délimitées constituées d'hépatocytes ayant une disposition trabéculaire ou tubuleuse. Ces derniers présentent une anisocaryose, une anisocytose, et un nucléole volumineux. L'ensemble est évocateur d'un adénocarcinome, viroinduit dans le cas présent. En ce qui concerne les capillaires, seuls les œufs sont observés, les tissus et organes parasitaires, tels que les cordons génitaux, ont disparu.

\section{DISCUSSION}

Les parasites hépatiques de la marmotte ne sont pas fréquents (Anderson et al., 1990; Rausch, 1977; Roth et al., 1991; Sabatier, 1989). Ils n'avaient pas encore été identifiés sur des marmottes importées et utilisées comme modèle de laboratoire.

La prévalence de l'infestation par Taenia mustelae, dont les hôtes définitifs sont divers Mustélidés, semble faible (Roth, King et Tennant, 1991). Roth, en 1991, a trouvé que 10 marmottes américaines séronégatives visà-vis du WHV sur 128 présentaient des granulomes hépatiques d'origine parasitaire (Roth, King et Tennant, 1991). Seuls trois ont été identifiés : un était dû à Calodium hepaticum, un autre à Taenia mustelae, le dernier à Ackertia marmotae (Nematoda : Filarioidea). Deux autres parasites peuvent être observés dans le foie de marmottes : Taenia crassiceps et Echinococcus multilocularis (Albert, Chapman et Pursley, 1981; Albert et al., 1972; Anderson et al., 1990; Fatzer, Horning et Fankhauser, 1976 ; Logachev et Bat'kaev, 1979). Taenia crassiceps est un parasite fréquent de la marmotte, avec une prévalence moyenne de 2,6 à 7,3\% selon les auteurs, mais la localisation habituelle des cysticerques est sous-cutanée (Albert, Chapman et Pursley, 1981; Albert et al., 1972; Anderson et al., 1990; Fatzer, Horning et Fankhauser, 1976). Les cysticerques peuvent cependant avoir des localisations viscérales : intrahépatiques (Anderson et al., 1990), pulmonaires (Anderson et al., 1990), ou nerveuses (Fatzer, Horning et Fankhauser, 1976). Echinococcus multilocularis a été observé dans le foie de marmotte du Kazaksthan, Marmota bobac, à 6 reprises au cours de 130 autopsies (Logachev et Bat'kaev, 1979). La diagnose différentielle entre les larves de Taenia mustelae et d'Echinococcus multilocularis repose essentiellement sur l'observation d'un cysticerque ou de vésicules contenant plusieurs protoscolex dans le cas de l'échinocoque. La diagnose ne doit pas uniquement reposer sur l'observation des crochets, lesquels sont de taille et d'aspect semblables chez ces deux parasites. L'extension de l'échinococcose multiloculaire aux États-Unis (Hildreth, Jonhson et Kazacos, 1991), et la réceptivité probable de Marmota monax à ce cestode, nécessitent la recherche de ce parasite lors de la réalisation des autopsies.

Calodium hepaticum est un parasite peu spécifique, de répartition cosmopolite (Gevrey et Chirol, 1978; Singleton et al., 1991). La prévalence de l'infestation des marmottes par ce nématode n'est pas connue. Les marmottes parasitées s'infestent en consommant les œufs présents dans le milieu extérieur. Ces derniers sont le plus souvent éliminés et dispersés, de façon passive, par les carnivores, tels les Mustélidés, qui ont consommé des rongeurs parasités. Par conséquent, ce parasitisme est uniquement observé chez des animaux sauvages capturés. Calodium hepaticum est un parasite zoonosique, qui peut se localiser chez l'homme, comme chez ces hôtes habituels, dans le parenchyme 


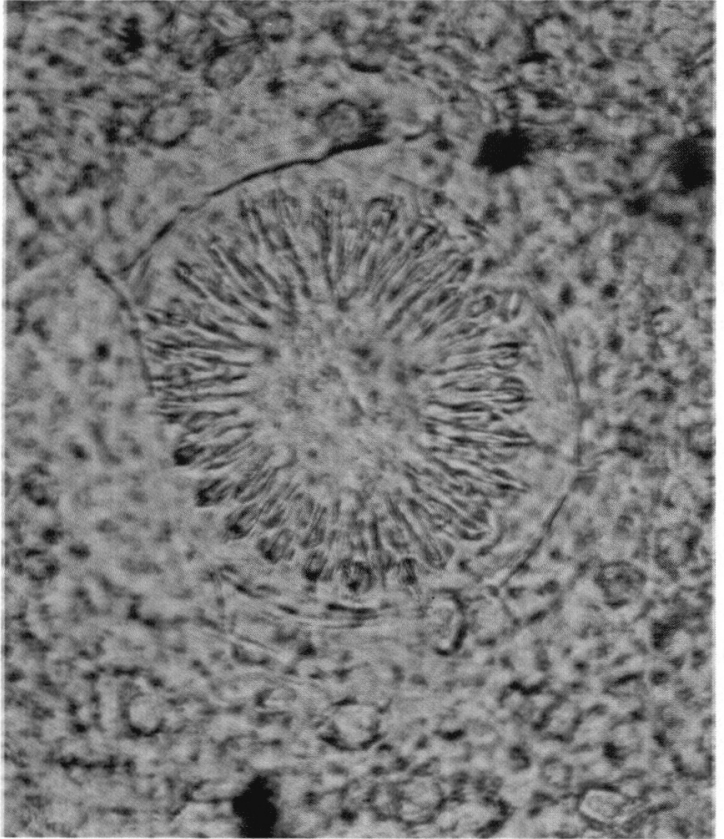

Photo 1: Scolex de Taenia mustelae. Examen direct (obj. $\times 10)$.

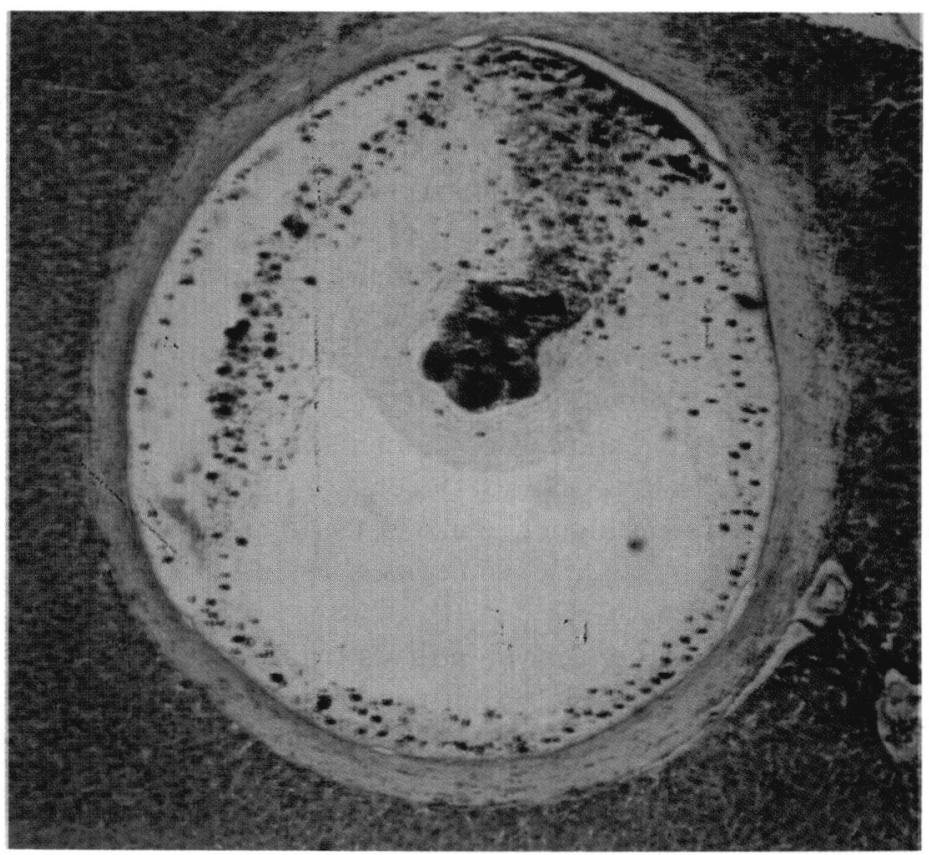

Photo 2 : Coupe d'un cysticerque de T. mustelae au sein du parenchyme hépatique. Coloration HE (obj. $\times 10)$.

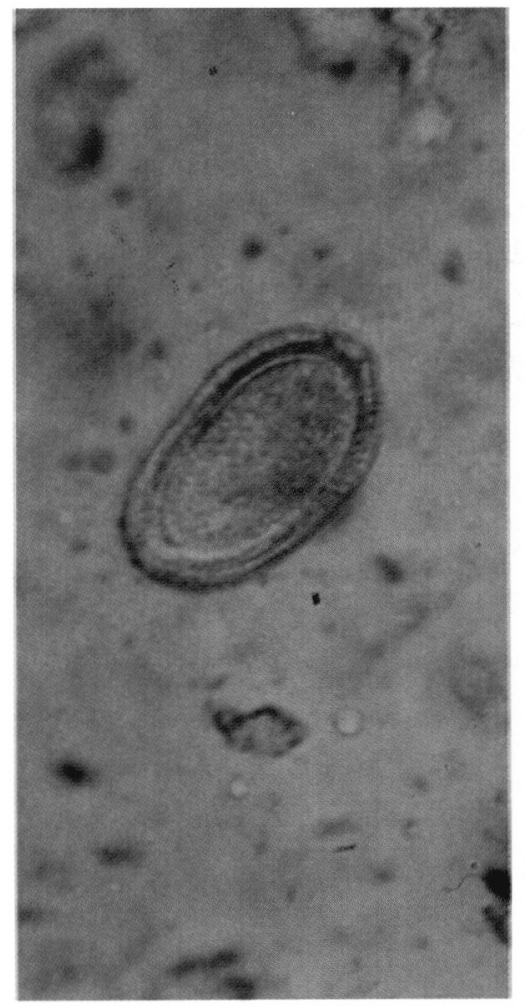

Photo 3: Cuf de Calodium hepaticum (obj. $\times$ 40)

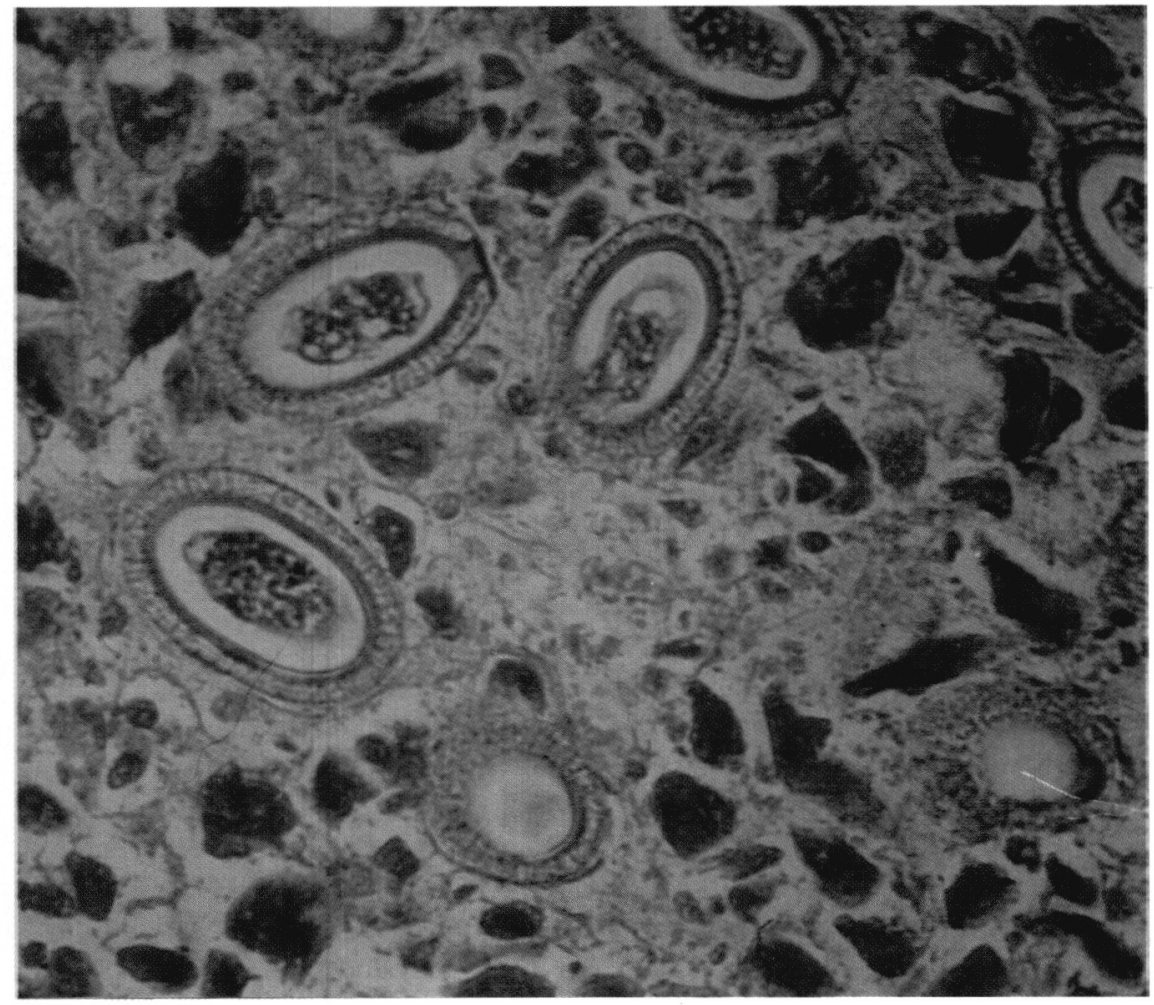

Photo 4 : Coupe histologique du parenchyme hépatique. CEufs de Calodium hepaticum. Coloration $\mathrm{HE}(\mathrm{obj} . \times 40)$. 
hépatique, jamais dans le tractus digestif. L'infestation se fait par consommation d'œufs embryonnés présents dans le milieu extérieur. A l'instar des carnivores, sans héberger le parasite, la consommation de rongeurs parasités peut entraîner l'élimination d'œufs de $C$. hepaticum dans les selles humaines. Ces derniers sont alors à distinguer d'autres oufs capillaires pouvant s'y trouver, notamment les oufs de Eucoleus aerophilus (syn.Capillaria aerophila) $(60-75 \times 35-40 \mu \mathrm{m})$ ou de Capillaria philippinensis $(40 \times 20 \mu \mathrm{m})$.

Ces parasitoses hépatiques, notamment la capillariose, induisent des lésions qu'il est nécessaire de connaître de façon à les distinguer, dans le cas des modèles expérimentaux d'hépatite virale, des lésions induites par le virus WHV.

La question de l'inter-action entre les parasites à localisation hépatique et l'infection par le WHV se pose, ce virus étant naturellement présent dans les populations sauvages de marmottes. Les parasites pourraient favoriser l'installation du virus ou le passage d'un état de portage latent au déclenchement de l'hépatite. Inversement, les marmottes séropositives, développant ou non une hépatite viro-induite, pourraient être plus réceptives à l'infestation parasitaire que les marmottes séronégatives. Le recueil de données sur le parasitisme chez les marmottes, sauvages et utilisées en laboratoire, sera nécessaire pour pouvoir répondre à ces questions. Anderson, en 1991, n'a pas mis en évidence d'interaction entre l'infection par le WHV et l'infestation par Taenia crassiceps, la prévalence de l'infestation parasitaire n'étant pas significativement différente entre les populations séronégatives et séropositives. De telles études méritent d'être conduites avec les parasites à localisation hépatique.

\section{REMERCIEMENTS}

$\mathrm{R}$ emerciements au Pr. J. Gastellu pour la réalisation et l'interprétation des examens histologiques.

\section{RÉFÉRENCES}

ABULADZE K.I. Taeniata of animals and man and diseases caused by Them. Essentials of Cestodology. Volume IV. Ed Skrjabin K.I.. Israel Program for Scientific Translations, 1970, $549 \mathrm{p}$.

Albert T.F. \& Chapman J.A. (ed.) Pursley-D. Massive infection of tapeworm larvae (Taenia crassiceps) in woodchucks (Marmota monax). Worldwide furbearer conference proceedings, 3-11 August, 1980, Frostburg, Maryland, USA. Volume II, 1981, 670-677.

Albert T.F., Schueler R.L., Panuska J.A. \& Inglng A.L. Tapeworm larvae (Taenia crassiceps) in woodchucks. Journal of the American Veterinary Medical Association, 1972, 161, 648-651.

Anderson W.I., Scott D.W., Hornbuckle W.E., King J.M. \& TEnnant B.C. Taenia crassiceps infection in the woodchuck: a retrospective study of 13 cases. Veterinary Dermatology, 1990, 1, 85-92, 14.

BANCROFT T.L. On the whipworm of the rat's liver. Journal and Proceedings of the Royal Society of New South Wales, 1893, 27, 86-90.

Fatzer R., Horning B. \& Fankhauser R. Taenia crassiceps cysticercus (Cysticercus longicollis) in the central nervous system of the Alpine marmot (Marmota marmota).Verhandlungsbericht des XVIII Internationalen Symposiums uber die Erkrankungen der Zootiere, Innsbruck, 1976, 111-113.

Gevrey J. \& Chirol C. A propos d'un cas de capillariose à Capillaria hepatica observé dans un élevage de lapins croisés Garenne. Revue de Médecine Vétérinaire, 1978, 129, 1019-1026.

Logachev E.D., BAT'KaEv A.I. Marmota bobac, a new intermediate host of Echinococcus multilocularis. Voprosy Prirodnoi Ochagovosti Boleznei, 1979, 10, 133-141.

HiLdReth B., Jonhson M.D. \& Kazacos K.R. Echinococcus multilocularis: a zoonosis of inceasing concern in the United States. The Compendium, 1991, 13, 727-741.

Rausch R.L. The specific distinction of Taenia twitchelli Schwartz, 1924 from T. martis (Zeder, 1803) (Cestoda: Taeniidae). Excerta parasitologica en memoria del Doctor Eduardo Caballero y Caballero, 1977, 357-366.

Roth L., King J.M. \& Tennant B.C. Hepatic lesions in woodchucks (Marmota monax) seronegative for woodchuck hepatitis virus. Journal of Wildlife Diseases, 1991, 27, 281287.

SABATIER B. Les parasites de la Marmotte Alpine : étude dans les Alpes françaises et synthèse bibliographique. Thèse de Doctorat Vétérinaire, 1989, Lyon, 178 p.

Singleton G.R., Spratt D.M., Barker S.C. \& Hodgson P.F. The geographic distribution and host range of Capillaria bepatica (Bancroft) (Nematoda) in Australia. International Journal for Parasitology, 1991, 21, 945-957.

Reçu le 27 décembre 1995 Accepté le 26 mars 1996 